\title{
Study the Optical Properties of Iron- Free Magnetic Lens
}

\author{
Roaa T. Abdulla \\ Department of Physics, College of Science, University of Al-Nahrain, Baghdad-Iraq. \\ E-mail: roaa.tahseen83@gmail.com.
}

\begin{abstract}
Attention has been concentrated on the effect of the current density and lens dimensions on iron-free magnetic lens design. A magnetic field has been calculated for a such magnetic lens for different values of current density. Also, the radial and spiral distortion aberration coefficients have been computed. Results have clearly shown that the low aberration coefficients can get at current density of high value and the ratio $S / D$ of the system within the rang $0.5 \leq S / D \leq 2$.
\end{abstract}

Keywords: Iron-free magnetic lens, Current density, Spiral distortion, radial distortion.

\section{Introduction}

All electron optical lenses have aberration, which limit the resolution of the image. There are two distinct factors that limit the performance of the magnetic lens. The first the maximum axial field flux density. The second is the maximum current density in the coil [1]. The limiting factor for the axial flux distribution $B z$, of a given magnetic lens, is the current density $\sigma$ that can be supported by the energizing coil. Hence, for a specific ratio (D2/D1) of the outer to inner diameter, and $S / D_{m}$, the ratio of the axial thickness to the mean diameter, the actual size of the coil and hence of a lens of given shape, will be decided by the current density in the winding, since in electron optics the excitation NI is usually a given quantity. For better resolution, the required lens performance can be achieved only by the use of iron polepieces separated by an extremely small gap and bore, usually on the order of a millimeter it may be useful to consider the current density as a technological parameter to improve the EM performance [2]. The resolution of both single and double polepiece lenses is approximately the same. Moreover, an improvement in the resolution may not be worthwhile in practice, for a current density $\sigma$ greater than about $10^{5} \mathrm{~A} / \mathrm{cm}^{2}$ [3]. An iron-free coil offers new possibilities for overcoming these disadvantages. In principle, an iron-free objective lens provides a lower aberration coefficient at very much higher current density $\sigma$ than is possible with an iron-polepieces lens [4]. One advantage of iron-free coils is their size reduction with respect to the iron circuit lenses. It is therefore desirable to describe some recent investigations of iron-free objective lenses that offer the possibility of developing electron optical instruments, both with and without the use of superconducting windings [5]. The computation in this article has been carried out for the iron-free magnetic lens. Also, to study the favorable practical current density regions suitable for iron-free magnetic lens.

\section{Theory \\ a- Iron-free lenses}

Although iron-free lenses have some technical problems, they may still have a role to play when the need for space around the specimen is paramount [6]. Tap coils made from copper insulated by a thin Maylar tape can be constructed with high symmetry and produce high flux density when operated at current densities of the order of $10^{4} \mathrm{~A} / \mathrm{cm}^{2}$. Much higher current densities can be achieved with super-conducting windings, which will become increasingly important for future highvoltage, high-resolution microscopes [7].

The axial field distribution of an iron-free coil can be calculated analytically. This greatly simplifies the analysis compared with that needed for iron pole-piece lenses. Consider a coil Fig.(1) of rectangular cross section, with outer radius $\mathrm{R} 2$, inner radius $\mathrm{R} 1$ and thickness s. the axial flux density $\mathrm{B}(\mathrm{z})$ of an iron-free coil is given by [5]:

$$
\begin{aligned}
& \mathrm{B}(\mathrm{z})=\frac{\mu_{\mathrm{o}} N I}{2 L S}\left(\left(\frac{\mathrm{S}}{2}-Z\right) \ln \frac{R 2+\sqrt{\left(R 2^{2}-\left(\frac{s}{2}\right)^{2}\right)}}{R 1+\sqrt{\left(R 1^{2}-\left(\frac{s}{2}\right)^{2}\right)}}\right. \\
& \left.+\left(\frac{S}{2}+Z\right) \ln \frac{R 2+\sqrt{\left(R 2^{2}+\left(\frac{s}{2}\right)^{2}\right)}}{R 1+\sqrt{\left(R 1^{2}-\left(\frac{s}{2}\right)^{2}\right)}}\right)
\end{aligned}
$$


where, $\mu 0=4 \pi * 10^{-7} \mathrm{H} / \mathrm{m}$ and $\mathrm{L}=\mathrm{R} 2-\mathrm{R} 1$. $\mathrm{R} 1, \mathrm{R} 2, \mathrm{z}$ and $\mathrm{s}$ are in meters, NI is in ampereturns and $\mathrm{B}(\mathrm{z})$ is in teslas.

Since $\sigma \mathrm{A}=\mathrm{NI}$, where $\sigma$ is the current density and $A=r(R 2-R 1) S=r S$, where $\gamma$ is the packing factor $(r=0.9$ for copper type windings), the axial flux density $\mathrm{B}(\mathrm{z})$ is takes the form:

$\mathrm{B}(\mathrm{z})=\frac{\mu \sigma_{\circ}}{2}\left(\left(\frac{\mathrm{S}}{2}-Z\right) \ln \frac{R 2+\sqrt{\left(R 2^{2}-\left(\frac{s}{2}\right)^{2}\right)}}{R 1+\sqrt{\left(R 1^{2}-\left(\frac{s}{2}\right)^{2}\right)}}\right.$

$+\left(\frac{\mathrm{S}}{2}+Z\right) \ln \frac{R 2+\sqrt{\left(R 2^{2}+\left(\frac{s}{2}\right)^{2}\right)}}{\left.R 1+\sqrt{\left(R 1^{2}-\left(\frac{s}{2}\right)^{2}\right)}\right)}$

Peak value $\mathrm{B}_{0}$ of the axial flux density can be expressed as:

$\mathrm{B}_{0}=\frac{\mu_{o} s}{2}\left(\ln \frac{R 2+\sqrt{\left(R 2^{2}+\left(\frac{s}{2}\right)^{2}\right)}}{R 1+\sqrt{\left(R 1^{2}+\left(\frac{s}{2}\right)^{2}\right)}}\right) \cdot \sigma$

There for, the maximum axial flux density $\mathrm{B}_{0}$ of an iron-free coil varies with $\sigma$, in other words the aberration of the iron-free coils varys as $\sigma$ proportional to $\sigma^{-1}$.

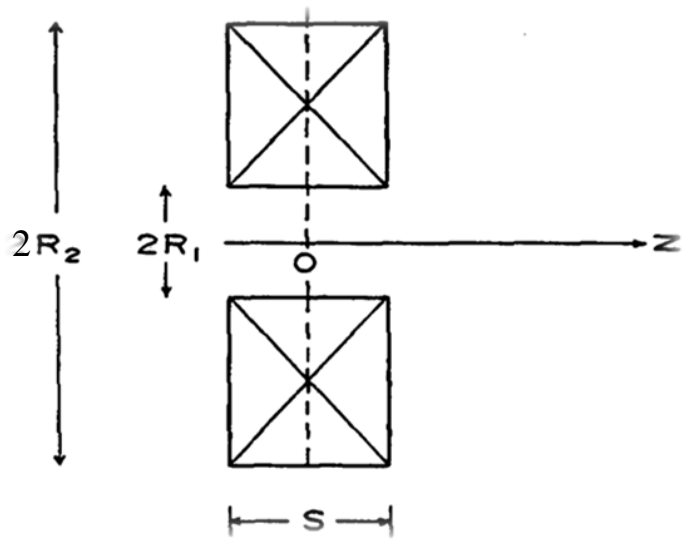

Fig.(1) An axialy symmetric iron free coil of rectangular cross section :inner radius $R_{1}$; outer radius $R_{2}$;coil thickness $S$; mean radius $R_{m}=\left(R_{1}+R_{2}\right) / 2,[1]$.

\section{b-Radial and Spiral distortion aberration}

Projection system can and usually does introduce distortion into the image. This sort of aberration detracts only from faithfulness, but not from the sharpness of image [8]. The spiral and radial distortion aberration coefficients of an axially symmetric magnetic optical element are given by [9]:

$$
\begin{aligned}
& D s p(V r)=\left(\frac{1}{16 \cdot V_{r}}\right) \cdot\left(2 \cdot \frac{q}{m q \cdot V_{r}}\right)^{1 / 2} * \\
& \int_{-\infty}^{\infty} B(z) \cdot\left[\left(3 \cdot \frac{q}{8 \cdot m q}\right)+B(z)^{2}+V r \cdot\left(\frac{Y d}{Y}\right)^{2}\right] \cdot Y^{2} d z \cdot . \\
& \operatorname{Drad}(V r)=\left(\frac{3}{8 \cdot p^{2}}\right) \cdot\left(2 \cdot \frac{q}{16 \cdot m q \cdot V_{r}}\right)^{1 / 2} * \\
& \int_{-\infty}^{\infty}\left[B(z)^{2}+\left(3 \cdot \frac{q}{8 \cdot m q \cdot V r}\right) \cdot B(z)^{4}-B(z) \cdot\left(\frac{Y^{\prime}}{Y}\right)^{2}\right] \cdot Y^{3} \cdot X d z
\end{aligned}
$$

where $\mathrm{X}$ and $\mathrm{Y}$ are two independent solutions of paraxial- ray equation with an initial condition depending on the operation modes, the prime denote derivative with respect to $\mathrm{z}, \mathrm{mq}$ is electron mass, $\mathrm{V}_{\mathrm{r}}$ is relativistic corrected accelerating voltage, $\mathrm{q}$ is electron mass and $\mathrm{fp}$ is the projector focal length.

\section{Results}

For the values of current density $\sigma=(5000,25000,50000,100000)$ (in unit $\mathrm{A} / \mathrm{cm}^{2}$, the axial magnetic field distribution for iron-free magnetic lens have been computed and the resultant curves are plotted in Fig.(2). The values of the inner diameter $\mathrm{D}_{1}$, outer diameter $\mathrm{D}_{2}$ and coil thickness $\mathrm{S}$ are maintained fixed at $2.7 \mathrm{~mm}$, $54.5 \mathrm{~mm}$ and $17.16 \mathrm{~mm}$ respectively. It is clear that $\mathrm{B}_{\max }$ increases as the values of current density $\sigma$ is increasing.

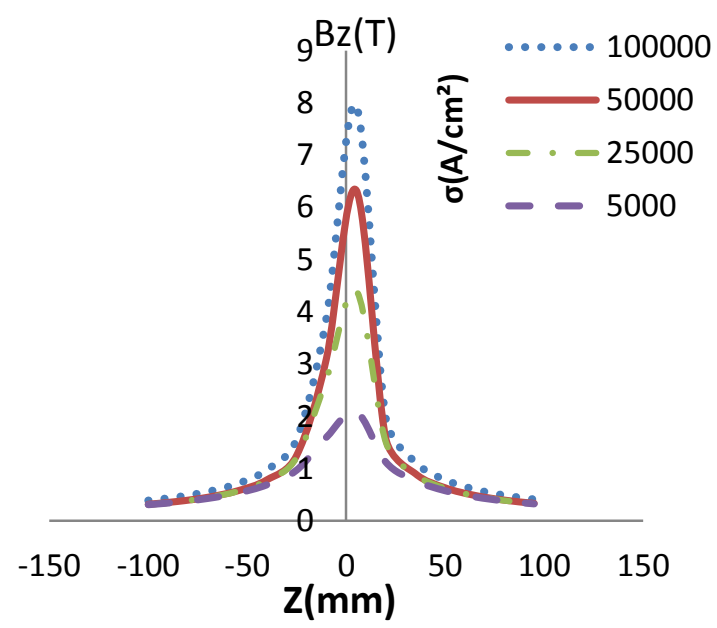

Fig.(2) The axial flux density $B(z)$

distribution of an iron-free magnetic lens for different values of current density. 
Fig.(3) and Fig.(4) shows a log-log plot of the of spiral and radial distortion aberration coefficients respectively as a function current density $\sigma$ with different value of ratio S/Dm. This kind with plot shows the variation of above coefficient more clear than a linear plot can. It is clear that the spiral and radial distortion aberration coefficients decrease with increasing current density. Fig.(3) show the minimum value of $\mathrm{Dsp}=0.02 \mathrm{~mm}$ at $\mathrm{S} / \mathrm{Dm}=0.5$ and current density $\sigma=4 * 10^{6} \mathrm{~A} / \mathrm{cm}^{2}$. Fig.(4) show the minimum value of $\mathrm{Drad}=0.0132$ at $\mathrm{S} / \mathrm{Dm}=0.5$ and current density $\sigma=4 * 10^{6} \mathrm{~A} / \mathrm{cm}^{2}$. It is clear that the iron free magnetic lens with $\mathrm{S} / \mathrm{Dm}=0.5$ has the smallest both Drad and Dsp value.

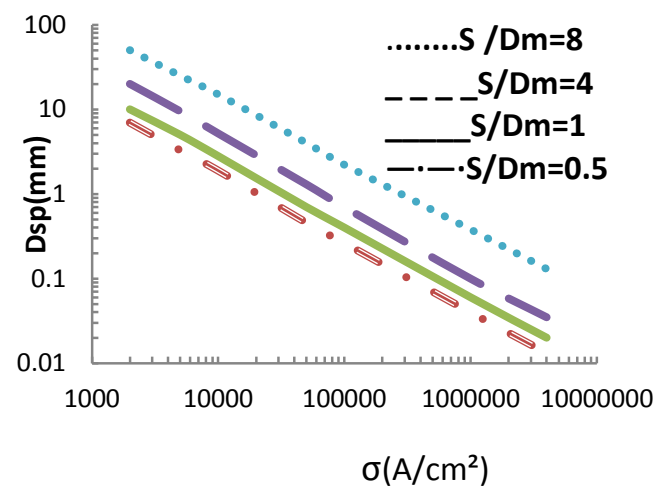

Fig. (3) The variation of the spiral distortion coefficient of iron-free magnetic lense with current density $\sigma(z=0, V=1 M V)$.

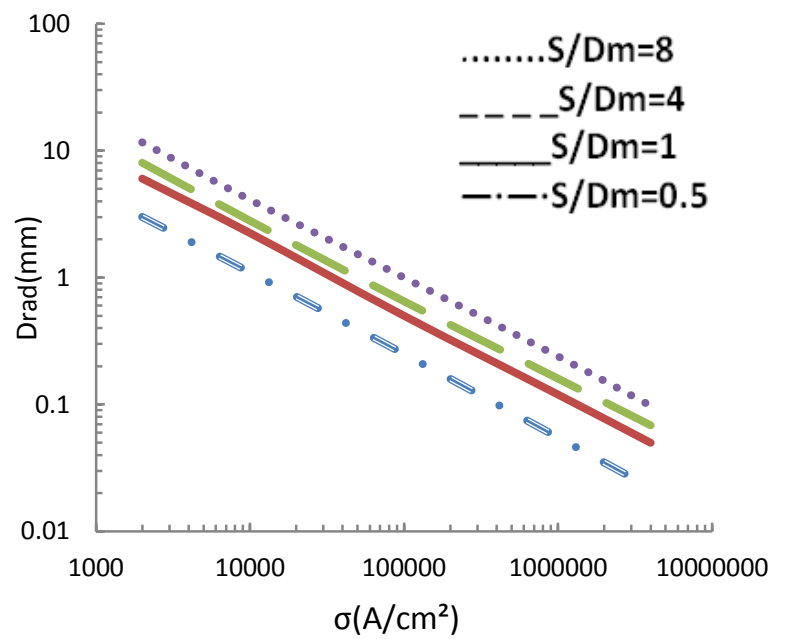

Fig. (4) The variation of the radial distortion coefficient of iron-free magnetic lense with current density $\sigma(z=0, V=1 M V)$.

Variation of radial and spiral distortion aberration coefficients together with lens geometry S/Dm is shown in Fig.(5) when the ratio $\mathrm{D} 2 / \mathrm{D} 1=58.5 \mathrm{~cm} / 3 \mathrm{~cm}=19.5$. The behavior of radial distortion aberration coefficient has the same behavior of spiral distortion aberration coefficient However, it should be noted that the radial and spiral distortion decreases slowly with increasing the ratio S/Dm especially in the value of S/Dm less than 1 , while for the value of $S / D_{m}$ approximately greater than 1 , the coefficients begin to increases. From Fig.(5) one can see that the minimum value of $\mathrm{Dsp}=0.26 \mathrm{~mm}$ at lens geometry $\mathrm{S} / \mathrm{D}_{\mathrm{m}}=0.5$ and the minimum value of Drad $=0.04 \mathrm{~mm}$ at lens geometry $\mathrm{S} / \mathrm{D}_{\mathrm{m}}=1$.

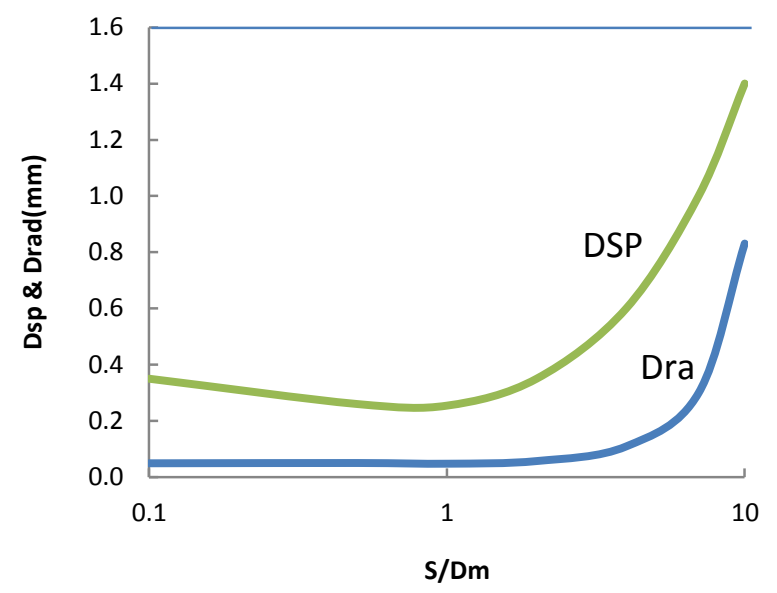

Fig. (5) The spiral and radial distortion coefficient together of iron-free magnetic lens as a function of coil geometry $S / D m$ when $D 2 / D 1=36.6(z=0)$.

Fig.(6) show how the radial and spiral distortion aberration coefficients varies with lens geometry S/Dm Calculations for Dsp and Drad for Fig.(6) are executed at the ratio $\mathrm{D} 2 / \mathrm{D} 1=55 \mathrm{~cm} / 1.5 \mathrm{~cm}=36.6$. The behavior of radial and spiral distortion coefficient in Fig.(6) has the same behavior of spiral distortion coefficient in Fig.(5). However, it should be noted that From figure the minimum value of $\mathrm{Dsp}=0.82 \mathrm{~mm}$ at lens geometry $\mathrm{S} / \mathrm{D}=1.5$ and the minimum value of $\mathrm{Drad}=0.1 \mathrm{~mm}$ at lens geometry $\mathrm{S} / \mathrm{D}=0.5$.the results from Fig.(5) and (6) are agreement with the conclusion drawn by Riecke(1) that for the design of an optimum objective lens, the ratio S/D of the system should be chosen within the rang $0.5 \leq \mathrm{S} / \mathrm{D} \leq 2$. It is important to mention that the Calculations for Dsp and Drad for both Figures (5) and (6) are executed at constant values of current density $\sigma=2 * 10^{4}\left(\mathrm{~A} / \mathrm{cm}^{2}\right)$ and $\mathrm{V}=1 \mathrm{MV}$. 


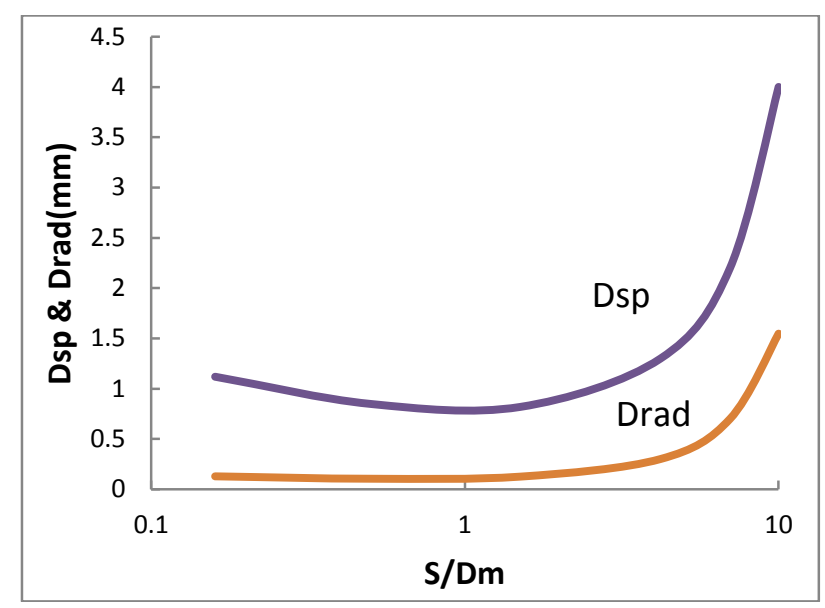

Fig. (6) The spiral and radial distortion coefficient together of iron-free magnetic lens as a function of coil geometry $S / D m$ when $D 2 / D 1=36.6(z=0)$.

\section{Conclusions}

The current density $\sigma$ is effectively the chief factor that limits the design and hence the electron- optical properties of magnetic lens. The dimensions and optical properties of the lens can be determined by choosing the value of $\sigma$ corresponding to desire minimum aberration. So, the minimum aberration can get at $\sigma$ of high value was taken and the coil geometry ratio $S / D_{m}$ less or equal to unity.

It appears from the present work it is possible to use any one of these results to find an optical system with small values of any radial and spiral distortion coefficient.

\section{References}

[1] Alamir, A.S.A; "Magnetic electron lenses performance"; Optik, 11, 507-510, 2001.

[2] Alamir, A.S.A; "A study on effect of current density on magnetic lenses"; Optik, 2, 85-88, 2003.

[3] Al-Nakeshili, I. S.; Juma, S. A.; and Mulvey, T.; "Electron Microscopy and nalysis"; Inst. Phys. Conf. Ser. 68, 475, 1984a.

[4] Alamir, A. S. A.; "Iron-free objective lenses"; J. phys. D. Appl. Phys. 25, 10391042, 1992.

[5] Alamir, A. S. A.; "High Voltage and High Current Density Objective Lens"; Chin. J. Phys. 38, 139-145, 2000.
[6] Hawkes P. W.; and Valdre, U.; "Superconductivity and Electron Microscopy"; J. Phys., E 309, 1977.

[7] Lefranc, G.; Knapek, E.; and Dietrich, I. "Superconducting Lens Design"; Ultramicroscopy 10, 111-123, 1982.

[8] Alamir, A. S. A.; "Spiral distortion of magnetic lenses with fields of the form $\mathrm{B}(\mathrm{z})$ $\alpha \mathrm{z}^{-\mathrm{n}} \mathrm{n}=2,3,4$ "; Optik 12, 525-528, 2003.

[9] Tsudo, K.; Harada, Y.; "Elimination of spiral distortion in electron microscopy using an asymmetrical triple pole-piece lens"; J. Phys. E: Sci. Instrum. 14, 955-960, 1981.

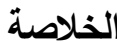

تم التركيز على تاثير كثافة الفيض المغناطيسي وابعاد

العدسة على تصميم العدسة المغناطيسية الخالية اقطابها من

الحديد. المجال المغناطيسي لهذه العدسة قد وجد باستخدام

قيم مختلفة من كثافة الفيض المغناطيسي وكذلك تم حساب

معاملات زيغي التشويه الثعاعي والتشويه الحلزوني. ان

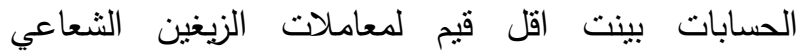

والحلزوني يمكن الحصول عليها عند القيم العالية لكثافة

الفيتض وعندما تكون قيم النسبة S/D ما بين

$.0 .5 \leq \mathrm{S} / \mathrm{D} \leq 2$ 